\title{
Magnon Damping by magnon-phonon coupling in Manganese Perovskites
}

\author{
Pengcheng Dai, ${ }^{1}$ H.Y. Hwang, ${ }^{2}$ Jiandi Zhang, ${ }^{1,}$. J. A. Fernandez-Baca, ${ }^{1}$ S-W. Cheong, ${ }^{2,3}$ \\ C. Kloc, ${ }^{2}$ Y. Tomioka, ${ }^{4}$ and Y. Tokura ${ }^{4,5}$ \\ ${ }^{1}$ Oak Ridge National Laboratory, Oak Ridge, Tennessee 37831-6393 \\ ${ }^{2}$ Bell Laboratories, Lucent Technologies, Murray Hill, New Jersey 07974 \\ ${ }^{3}$ Department of Physics and Astronomy, Rutgers University, Piscataway, New Jersey 08855 \\ ${ }^{4}$ Joint Research Center for Atom Technology (JRCAT), Tsukuba 305-8562, Japan \\ ${ }^{5}$ Department of Applied Physics, University of Tokyo, Tokyo 113-8656, Japan
}

(August 29, 2018)

\begin{abstract}
Inelastic neutron scattering was used to systematically investigate the spinwave excitations (magnons) in ferromagnetic manganese perovskites. In spite of the large differences in the Curie temperatures $\left(T_{C} \mathrm{~s}\right)$ of different manganites, their low-temperature spin waves were found to have very similar dispersions with the zone boundary magnon softening. From the wavevector dependence of the magnon lifetime effects and its correlation with the dispersions of the optical phonon modes, we argue that a strong magnetoelastic coupling is responsible for the observed low temperature anomalous spin dynamical behavior of the manganites.
\end{abstract}

PACS numbers: 72.15.Gd, 71.30.Kz, 61.12.Gz 
The elementary magnetic excitations (spin waves) in a ferromagnet can provide direct information about the itinerancy of the unpaired electrons contributing to the ordered moment. In insulating (local moment) ferromagnets, such excitations are usually well defined throughout the Brillouin zone and can be described by the Heisenberg model of magnetism [1]. On the other hand, metallic (itinerant) ferromagnets are generally characterized by the disappearance of spin waves at finite energy and momentum due to the presence of the Stoner (electron-hole pair) excitation continuum associated with the band structure and itinerant electrons in the system [2]. In the ferromagnetic manganese perovskites (manganites) $A_{0.7} B_{0.3} \mathrm{MnO}_{3}$ (where $A$ and $B$ are rare-earth and alkaline-earth elements respectively.) [3], the ferromagnetism and electric conductivity can be continually suppressed by different $A(B)$ substitutions until an insulating, charge-ordered ground state is stabilized [4]. Approaching this insulating ground state (with decreasing Curie temperature $T_{C}$ ), the low temperature spin-wave dispersions have been found to deviate [5] from the nearest-neighbor Heisenberg exchange Hamiltonian that has been successfully applied to the higher $T_{C}$ materials [6-9]. However, the microscopic origin for such deviation is unknown. Furthermore, spin waves for the lower $T_{C} A_{0.7} B_{0.3} \mathrm{MnO}_{3}$ have very similar spin-wave stiffness constants $(D)$, contrary to the expectation of a Heisenberg ferromagnet where $D$ is related to $T_{C}$ [10, 11]. Here we argue that the deviation from the simple Heisenberg Hamiltonian and the observation of magnon damping at large wavevectors are due to strong magneto-elastic (or magnon-phonon) interactions, consistent with the electron-lattice coupling [12] for the manganites in the proximity of the charge ordered insulating state.

We study $A_{0.7} B_{0.3} \mathrm{MnO}_{3}$ manganites because these materials exhibit a large resistivity drop that is intimately related to the paramagnetic-to-ferromagnetic phase transition at $T_{C}$ [3]. Due to the octahedral crystalline field, the $3 d$ energy level of the $\mathrm{Mn}$ ion in $A_{0.7} B_{0.3} \mathrm{MnO}_{3}$ is split into a low-lying $t_{2 g}$ triplet and a higher energy $e_{g}$ doublet. In 1951, Zener [13 postulated that the conductivity in these mixed valence systems was due to the simultaneous hopping of an $\left(e_{g}\right)$ electron (with electron transfer energy $t$ ) from $\mathrm{Mn}^{3+}\left(3 d^{4}\right)$ to the connecting $\mathrm{O}^{2-}$ and from the $\mathrm{O}^{2-}$ to the $\mathrm{Mn}^{4+}\left(3 d^{3}\right) e_{g}$ band; hence termed double-exchange (DE). 
Because all the electrons in the Mn $3 d$ levels are polarized by a strong intra-atomic exchange interaction $J_{H}$ (Hund-rule coupling), such hopping tends to align the spins of Mn ions on adjacent sites parallel [14. Thus, the DE mechanism provided a qualitative interpretation of coupled ferromagnetic ordering and electric conductivity [3] . However, recent theoretical work [15.16] suggests that DE mechanism alone cannot explain the temperature dependence of the resistivity near and above $T_{C}$. Additional interactions, such as a strong dynamical Jahn-Teller (JT) based electron-lattice coupling, are necessary to explain the magnitude of the resistivity drop across $T_{C}$. In this scenario, the JT distortion of the $\mathrm{Mn}^{3+} \mathrm{O}_{6}$ octahedra lifts the double degeneracy of $e_{g}$ electrons in $\mathrm{Mn}^{3+}$ and causes a local lattice distortion that may trap carriers to form a polaron. The formation of lattice polarons in the paramagnetic state leads to the localization of conduction band $\left(e_{g}\right)$ electrons above $T_{C}$ and hence the insulating behavior. On cooling below $T_{C}$ however, the growing ferromagnetic order increases the $e_{g}$ electron hopping (kinetic) energy $t$ and decreases the electron-lattice coupling strength sufficiently that metallic behavior occurs. The system crosses over from a polaron regime to a Fermi-liquid regime and the DE mechanism ultimately prevails in the low temperature metallic state [15].

The electron-lattice coupling described above is dynamical, i.e., it involves vibrational distortions of the oxygen octahedron around the $\mathrm{Mn}^{3+}$ site. However, the static lattice distortion, present because of the ionic size differences of $A$ and $B$ in $A_{0.7} B_{0.3} \mathrm{MnO}_{3}$, may also affect the electron hopping and the Curie temperature $T_{C}$. Therefore, systematic studies of the spin-wave excitations in $A_{0.7} B_{0.3} \mathrm{MnO}_{3}$ with decreasing $T_{C}$ and increasing residual resistivity [4] should reveal how the system evolves from an itinerant to a localized ferromagnet. Such investigation will also test to what extent the low temperature magnetic properties of $A_{0.7} B_{0.3} \mathrm{MnO}_{3}$ ferromagnets can be explained by the DE mechanism. For these purpose, we have characterized the low temperature spin dynamics of the ferromagnetic single crystals of $\mathrm{Pr}_{0.63} \mathrm{Sr}_{0.37} \mathrm{MnO}_{3}$ (PSMO), $\mathrm{La}_{0.7} \mathrm{Ca}_{0.3} \mathrm{MnO}_{3}$ (LCMO), and $\mathrm{Nd}_{0.7} \mathrm{Sr}_{0.3} \mathrm{MnO}_{3}$ (NSMO) which have approximately the same nominal carrier concentration but significantly different $T_{C} \mathrm{~S}$ and residual conductivity (see Figure 1). 
The temperature dependent resistivity $\rho(T)$ for these three samples is shown in Figure 1. The characteristic drop in $\rho$ coincident with $T_{C}$ is clearly seen to increase with decreasing $T_{C}$. At the same time the residual resistivity increases almost linearly with decreasing $T_{C}$, indicating that with increasing magnetoresistance effect the system becomes a worse metal at low temperatures. An interesting feature of $\rho(T)$ at low temperatures is that all three samples exhibit the same temperature dependence below $\sim 100 \mathrm{~K}$ when $\rho(T)$ is scaled to the residual value $\rho(0)$. The inset to Figure 1 illustrates this point.

The open symbols in Figure 2 summarize the spin-wave dispersions along the $[0,0, \xi]$, $[\xi, \xi, 0]$, and $[\xi, \xi, \xi]$ directions at $10 \mathrm{~K}$ for PSMO, LCMO, and NSMO [17. Clearly, the dispersions of these three manganites are remarkably similar at the measured frequencies, suggesting that the magnetic exchange coupling strength, derived from the hopping of the $e_{g}$ electrons between the $\mathrm{Mn}^{3+}$ and $\mathrm{Mn}^{4+}$ sites, depend only weakly on $T_{C}$. These results are in sharp contrast to the single-band DE model where the spin-wave dispersions are directly related to electronic bandwidth and hence $T_{C}$ [18 21]. In the strong coupling limit $\left(J_{H} / t \rightarrow \infty\right)$ of this model, the spin-wave dispersion of the ferromagnet is consistent with the nearest-neighbor Heisenberg Hamiltonian and $D$ should be proportional to the electron transfer energy $t$. Previous work has shown that such single-band DE model is adequate for describing the spin dynamics of the highest $T_{C}$ ferromagnetic manganites [6 9]. To estimate the spin-spin exchange coupling strength, we note that the low-frequency spin waves of $A_{0.7} B_{0.3} \mathrm{MnO}_{3}$ manganites LCMO [10, NSMO, and PSMO [11] are isotropic and gapless with a stiffness $D \approx 165 \mathrm{meV}^{-2}$. For a simple cubic Heisenberg ferromagnet with nearestneighbor exchange coupling $J, D=2 J S a^{2}$, where $S$ is the magnitude of the electronic spin at the magnetic ionic sites and $a$ is the lattice parameter. From the measured spinwave stiffness, one can calculate the exchange coupling strength $J$ and hence the expected dispersion for a simple nearest-neighbor Heisenberg ferromagnet. The solid lines in Figure 2 show the outcome of such calculation which clearly misses the measured spin-wave energies at large wavevectors.

Figure 3 shows typical constant-q scans along the $[0,0, \xi]$ and $[\xi, \xi, 0]$ directions for LCMO 
and NSMO. Most of the data are well described by Gaussian fits which give the amplitude, widths, and peak positions of the excitations. While the dispersion curves shown in Figure 2 are obtained by peak positions at different wavevectors, the amplitude and widths provide information about the damping and lifetime of the magnon excitations. The upper panel in Figure 3 displays the result along the $[0,0, \xi]$ direction and similar data along the $[\xi, \xi, 0]$ direction is shown in the bottom panel. It is clear that spin waves are significantly damped at large wavevectors. Although still relatively well-defined throughout the Brillouin zone in the $[0,0, \xi]$ direction for both compounds, the excitations are below the sensitivity of the measurements at wavevectors beyond $(0.25,0.25,0)$ reciprocal lattice units (rlu) along the $[\xi, \xi, 0]$ direction for NSMO.

To further investigate the wavevector dependence of the spin-wave broadening and damping, we plot in Figure 4 the intrinsic widths of the magnons along the $[0,0, \xi]$ direction. The full width at half maximum (FHWM) of the excitations $\Gamma$ shows a similar increase at wavevectors larger than $\xi \approx 0.3 \mathrm{rlu}$ for all three manganites [22]. To determine whether such broadening is due to the Stoner continuum, we note that at low temperatures, the spin moment of itinerant electrons of ferromagnetic manganites is completely saturated and the system is in the half-metallic state [23]. In this scenario of the DE model, there is complete separation of the majority and minority band by a large $J_{H}$. As a consequence, the Stoner continuum is expected to lie at an energy scale $\left(2 J_{H}\right)$ much higher than that of the spin-wave excitations [18, 19]. For this reason, the observed magnon broadening and damping for lower $T_{C}$ manganites are unlikely to be due to Stoner continuum excitations.

On the other hand, such behavior may be well understood if one assumes a new spinwave damping channel that is related to a strong coupling between the conduction band $\left(e_{g}\right)$ electrons and the cooperative oxygens in the Mn-O-Mn bond, analogous to that of a dynamic JT effect [12]. Although JT based electron-lattice coupling is known to be important for the metal-to-insulator transition at temperatures near and above $T_{C}$ [15,16, such coupling may also be important to understand the low temperature magnetic properties.

If electron-lattice coupling is indeed responsible for the observed spin-wave broadening 
and damping, one would expect the presence of such coupling in the lower $T_{C}$ samples that should be absent in the higher $T_{C}$ materials. Experimentally, there have been no reports of magnon-phonon coupling in the higher $T_{C} A_{0.7} B_{0.3} \mathrm{MnO}_{3}$ [6 8]. For the $\mathrm{DE}$ ferromagnet $\mathrm{La}_{0.8} \mathrm{Sr}_{0.2} \mathrm{MnO}_{3}\left(T_{C}=304 \mathrm{~K}\right)$, Moudden et al. [9] have measured the spin-wave, acoustic and optical phonon dispersions from the zone center to the zone boundary along the $[0,0, \xi]$ direction. Phonon branches were found to cross smoothly through the magnon dispersion, suggesting weak or no magnon-phonon hybridization. For the lower $T_{C}$ ferromagnet LCMO, infrared reflectivity spectra show three zone center $(\mathbf{q}=0)$ transverse optical $(\mathrm{TO})$ phonons located around $20.4 \mathrm{meV}, 42 \mathrm{meV}$, and $73 \mathrm{meV}$ [24]. These three phonon modes were identified as "external", "bending", and "stretching" modes which correspond to the vibration of the $\mathrm{La} / \mathrm{Ca}$ ions against the $\mathrm{MnO}_{6}$ octahedron, the bending motion of the $\mathrm{Mn}-\mathrm{O}-\mathrm{Mn}$ bond, and the internal motion of the Mn ion against the oxygen octahedron, respectively.

To search for possible magnon-phonon coupling in the lower $T_{C}$ manganites, we have measured selected optical phonons in the LCMO single crystal. Figure 2 shows two longitudinal optical (LO) phonon modes throughout the Brillouin zone as solid symbols. The metallic nature of the manganites at low temperature requires the collapse of the $\mathrm{LO}$ and TO splitting of the polar modes at $\mathbf{q}=0$. For LCMO, this means that the two LO modes observed by neutron scattering are likely to be the external and bending modes identified by infrared reflectivity [24]. At wavevectors larger than $0.3 \mathrm{rlu}$, the dispersions of these two phonon modes are remarkably close to those of the magnons along the $[0,0, \xi]$ and $[\xi, \xi, 0]$ directions, suggesting that the softening of the spin-wave branches in Figure 2 is due to magnon-phonon coupling.

In principle, the interaction between the magnetic moments and the lattice can modify spin waves in two different ways. First, the static lattice deformation induced by the ordered magnetic moments may affect the anisotropy of the spin waves. Second, the dynamic time-dependent modulations of the magnetic moment may interfere with the lattice vibrations, resulting in significant magneto-elastic interactions or magnon-phonon coupling. One possible consequence of such coupling is to create energy gaps in the magnon dispersion 
at the nominal intersections of the magnon and phonon modes. However, our spin-wave dispersion data in Figure 2 show no obvious evidence of any gap at the magnon and phonon crossing at $\xi \approx 0.3$ rlu. Alternatively, magnon-phonon coupling, present in all exchange coupled magnetic compounds to some extent, may give raise to spin-wave broadening [25]. In this scenario, the vibrations of the magnetic ions about their equilibrium positions affect the exchange energy through the spatial variation of the spin-spin exchange coupling strength, which in turn leads to spin-wave broadening at the magnon-phonon crossing points. Generally, one would expect such coupling to be strong for the lower $T_{C} A_{0.7} B_{0.3} \mathrm{MnO}_{3}$ manganites because of their close proximity to the charge-ordered insulating state [4]. This is exactly what is observed for these materials at $\xi \geq 0.3$ rlu. Constant-q scans in Figure 3 show significant broadening of the spin waves from $\xi<0.3$ to $\xi \geq 0.3$ rlu for LCMO and NSMO. Similarly, Figure 4 reveals that magnon widths increase considerably at wavevectors $\xi \geq 0.3$ rlu for all three manganites investigated, consistent with the expectation of a strong magnon-phonon hybridization [26].

We have discovered that spin-wave softening and broadening along the $[0,0, \xi]$ direction occur at the nominal intersection of the magnon and optical phonon modes in lower $T_{C}$ $A_{0.7} B_{0.3} \mathrm{MnO}_{3}$ manganites. This result strongly suggests that magneto-elastic coupling is important to the understanding of the low temperature spin dynamics of $A_{0.7} B_{0.3} \mathrm{MnO}_{3}$. In the lower doping canted ferromagnet $\mathrm{La}_{0.85} \mathrm{Sr}_{0.15} \mathrm{MnO}_{3}$ [27], much larger spin-wave broadening and damping have been found at low temperatures [28]. Although the magnon dispersion relation in that system appears to be consistent with the simple nearest-neighbor Heisenberg Hamiltonian, the observation of strong anisotropic spin-wave broadening is in sharp contrast to the expectation of the single-band DE model where magnons in the ground state are eigenstates of the system [28]. Therefore, it becomes clear that the single-band DE mechanism cannot describe the spin dynamics of $\mathrm{La}_{0.85} \mathrm{Sr}_{0.15} \mathrm{MnO}_{3}$ and lower $T_{C} A_{0.7} B_{0.3} \mathrm{MnO}_{3}$ manganites, not even in the low temperature ground state. To understand the extraordinary magnetic and transport properties of $A_{1-x} B_{x} \mathrm{MnO}_{3}$, one must explicitly consider the close coupling between charge, spin, and lattice degrees of freedom in these complex materials. 
We thank H. Kawano, W. E. Plummer, S. E. Nagler, H. G. Smith, and X. D. Wang for helpful discussions. This work was supported by the US DOE under Contract No. DE-AC05-96OR22464 with Lockheed Martin Energy Research Corporation and JRCAT of Japan. 


\section{REFERENCES}

* Present address: Department of Physics, Florida International University, University Park, Miami, Florida 33199.

[1] W. Heisenberg, Z. Phys. 49, 619 (1928).

[2] T. Izuyama et al., J. Phys. Soc. Jpn. 18, 1025 (1963).

[3] G. H. Jonker and J. H. Van Santen, Physica 16,337 (1950).

[4] H. Y. Hwang et al., Phys. Rev. Lett. 75, 914 (1995).

[5] H. Y. Hwang et al., Phys. Rev. Lett. 80, 1316 (1998).

[6] M. C. Martin et al., Phys. Rev. B 53, 14285 (1996).

[7] T. G. Perring et al., Phys. Rev. Lett. 77, 711 (1996).

[8] Y. Endoh and K. Hirota, J. Phys. Soc. Jpn. 66, 2264 (1997).

[9] A. H. Moudden et al., Physica B 241, 276 (1998).

[10] J. W. Lynn et al., Phys. Rev. Lett. 76, 4046 (1996).

[11] J. A. Fernandez-Baca et al., Phys. Rev. Lett. 80, 4012 (1998).

[12] J.-S. Zhou et al., Nature 381, 770 (1996); J. -S. Zhou and J. B. Goodenough, Phys. Rev. Lett. 80, 2665 (1998).

[13] C. Zener, Phys. Rev. 82, 403 (1951).

[14] P.W. Anderson and H. Hasegawa, Phys. Rev. 100, 675 (1955).

[15] A. J. Millis et al., Phys. Rev. Lett. 77, 175 (1996).

[16] H. Röder et al., Phys. Rev. Lett. 76, 1356 (1996).

[17] Although the samples were orthorhombic and twinned, we assume a cubic lattice $(a=$ 
$3.86 \AA$ ) for all three materials because the spectrometer could not resolve the effects of the twinning.

[18] N. Furukawa, J. Phys. Soc. Jpn., 65, 1174 (1996).

[19] T. Okabe, Prog. Theoretical Phys. 97, 21 (1997).

[20] T. A. Kaplan and S. D. Mahanti, J. Phys. Cond. Matt., 9, L291 (1997).

[21] X. D. Wang, Phys. Rev. B 57, 7427 (1998).

[22] To obtain $\Gamma$, we have deconveluted the spin-wave widths from the instrumental resolution calculated using the spin-wave dispersion at the measured wavevectors.

[23] J.-H. Park et al., Nature 392, 794 (1998).

[24] K. H. Kim et al., Phys. Rev. Lett. 77, 1877 (1996).

[25] S. W. Lovesey, Theory of neutron scattering from condensed matter (Clarendon press, Oxford, 1984).

[26] In principle, we should also observe a large spin-wave broadening at the magnon-phonon crossing points around $42 \mathrm{meV}$ along the $[\xi, \xi, 0]$ direction. However, we were unable to extract reliable magnon widths data due to the weakness of the spin-wave signal at these large energies (see Figure 3).

[27] H. Kawano et al., Phys. Rev. B 53, 2202 (1996).

[28] L. Vasiliu-Doloc et al., Phys. Rev. B 58, 14913 (1998). 


\section{FIGURES}

FIG. 1. Temperature dependence of the resistivity $\rho(T)$ for single crystals used in the neutron scattering experiments. The large drop in $\rho(T)$ corresponds to the Curie Temperature $\left(T_{C}\right)$ of our samples. They are $301 \mathrm{~K}, 238 \mathrm{~K}$, and $198 \mathrm{~K}$ for PSMO, LCMO, and NSMO, respectively. The inset shows the normalized resistivity $\rho(T) / \rho(0)$, it is clear that all three samples have the same $\rho(T) / \rho(0)$ temperature dependence below about $100 \mathrm{~K}$.

FIG. 2. Open symbols show magnon dispersions along the $[0,0, \xi],[\xi, \xi, 0]$, and $[\xi, \xi, \xi]$ directions for $\sim 30 \%$ manganites PSMO (open squares), LCMO (open circles), and NSMO (open down triangles) at $10 \mathrm{~K}$. The data for PSMO are from Ref. [5]. The solid line is a fit to a nearest-neighbor Hamiltonian assuming isotropic spin-waves for $\xi<0.1$. Full symbols show selected LO phonon modes collected along the reciprocal lattice directions as specified in the legend. The rapid decrease of the manganese magnetic form factor at these large wavevectors ensure that the scattering stem mostly from the lattice vibrations (phonons).

FIG. 3. Constant-q scans at selected wavevectors for LCMO and NSMO at $10 \mathrm{~K}$. The magnetic nature of the signal was confirmed by measuring the temperature and wavevector dependence of the scattering (see, for example, Figure 2 of Ref. [5]). The data were taken with PG as monochromator and analyzer at a final neutron energy of $E_{f}=13.6 \mathrm{meV}$. Analyzer turned background have been subtracted from the data. There is a dispersionless crystal electric field (CEF) level at $\sim 12 \mathrm{meV}$ from Nd for NSMO. The horizontal bars show the resolution along the scan direction. Solid lines are Gaussians fits to the data.

FIG. 4. The intrinsic magnon widths along the $[0,0,1+\xi]$ direction for PSMO, LCMO, and NSMO at $10 \mathrm{~K}$. Significant broadening of the magnon widths is seen at $\xi \approx 0.3 \mathrm{rlu}$. The arrow indicates the crossing point of magnon and phonon dispersions. Solid line is the guide to the eye. 


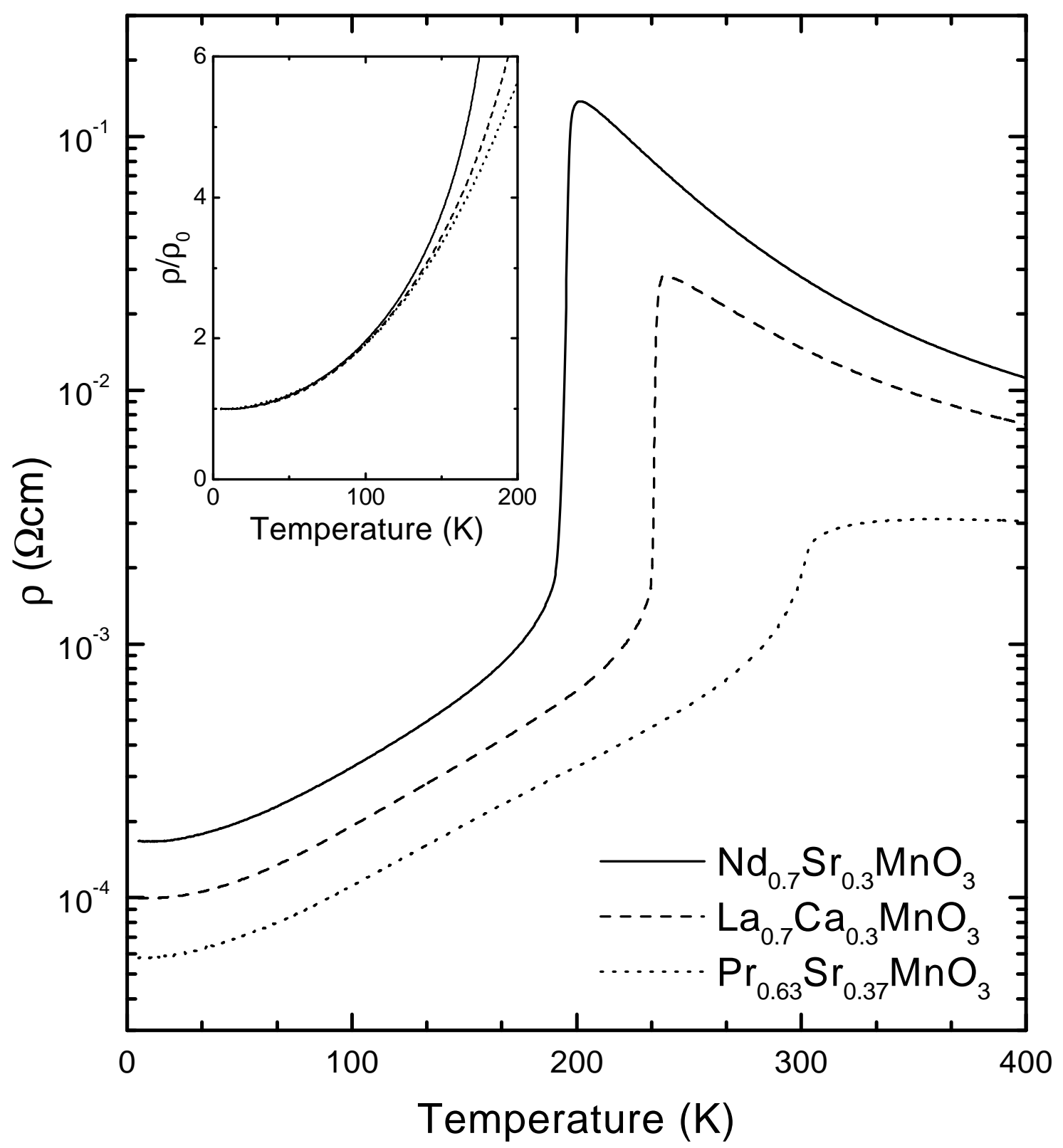

Figure 1, P. Dai et al. 


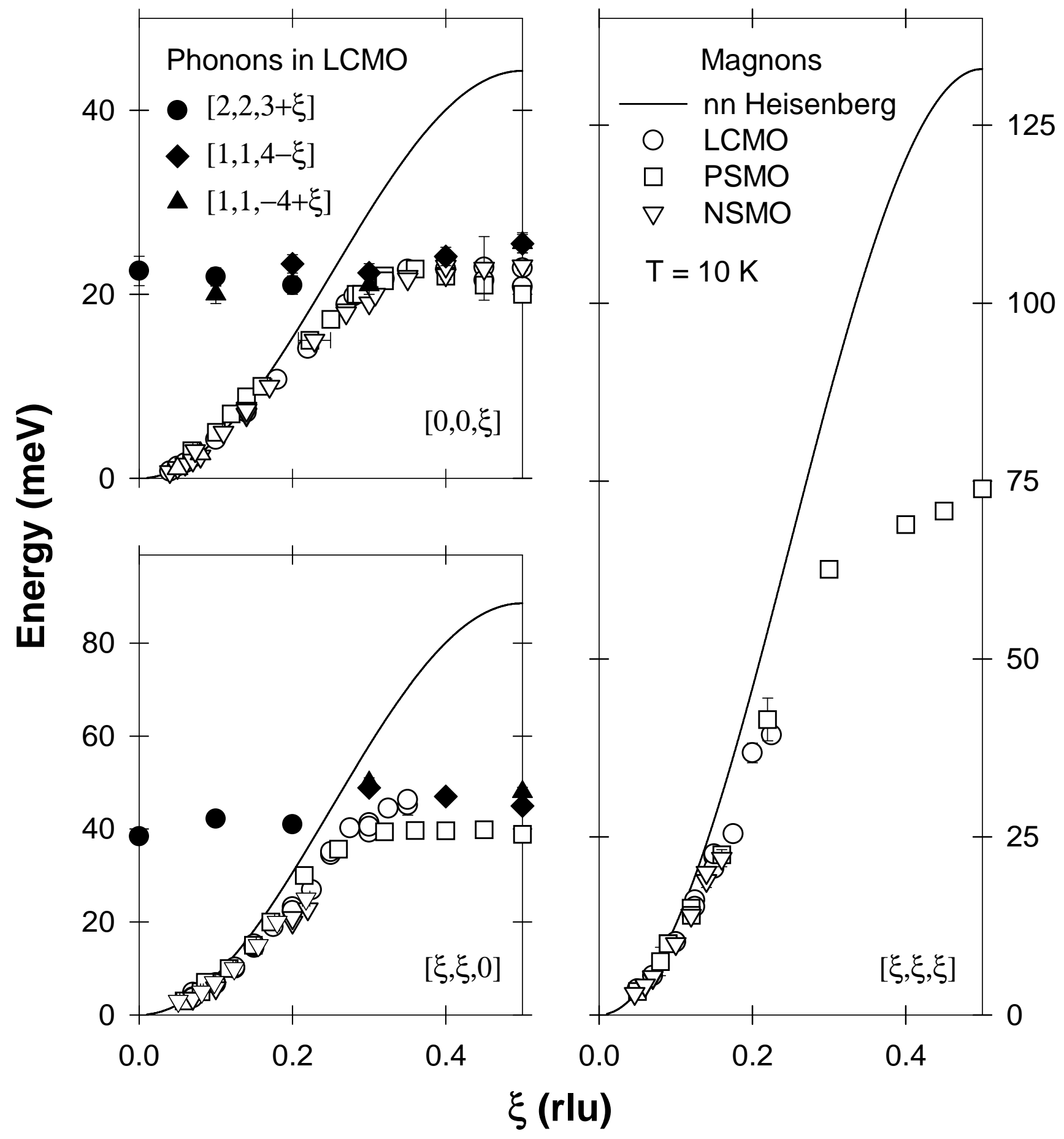

Figure 2

P. Dai et al. 


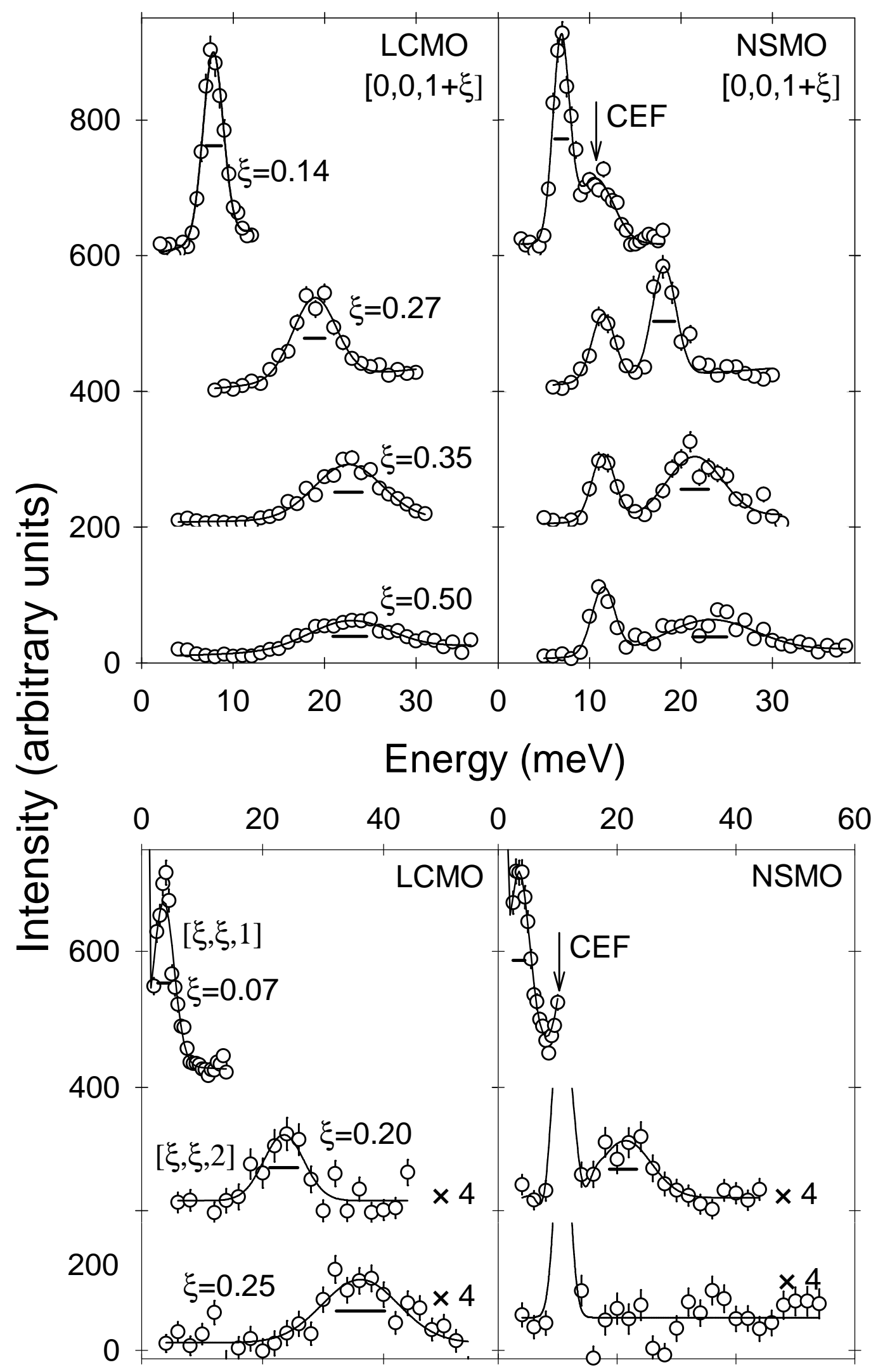

Figure 3

P. Dai et al. 


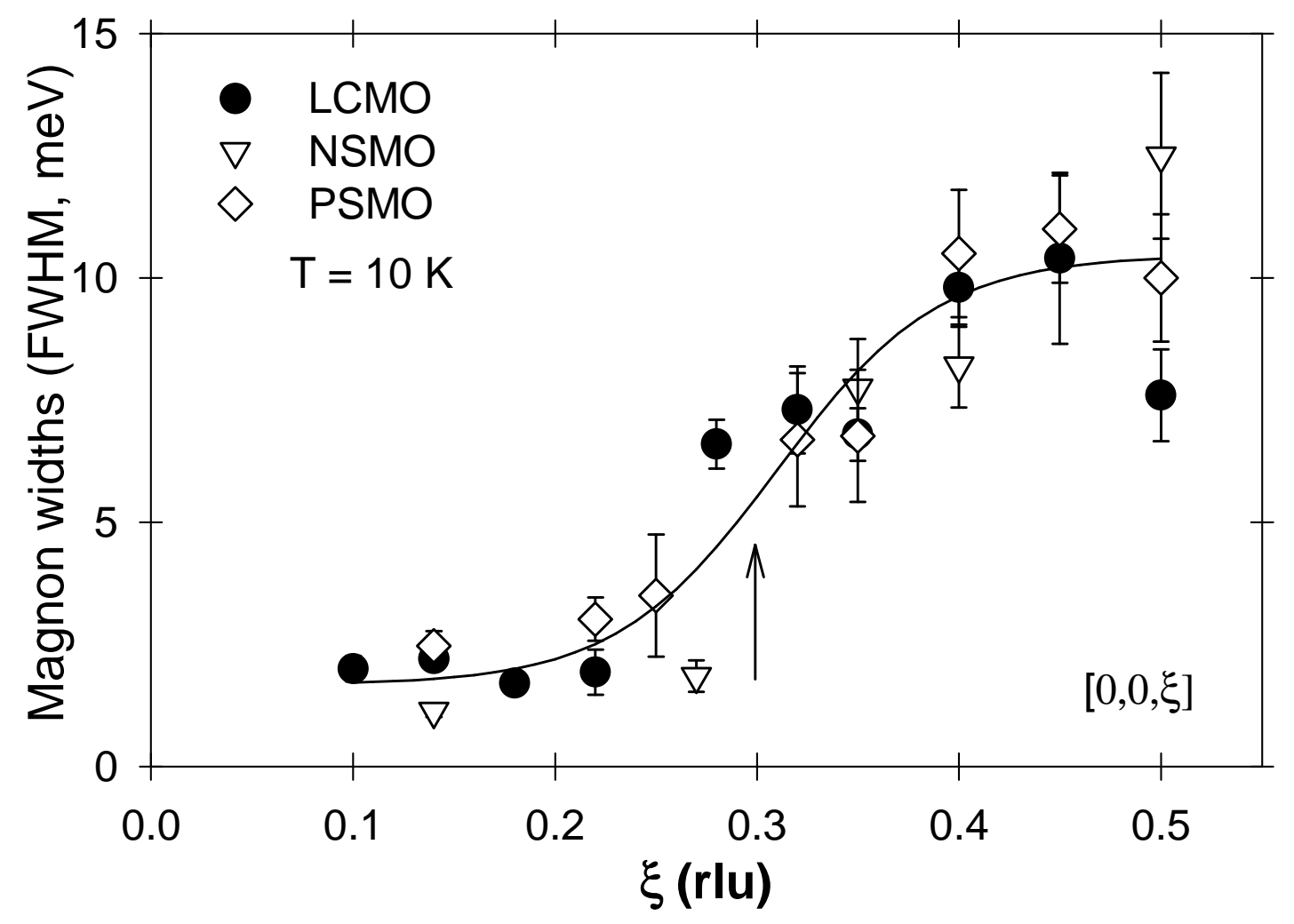

Figure 4

P. Dai et al. 